Ann. Biol. anim. Bioch. Biophys., I974, 14 (I), 87-104.

\title{
MÉTHODE D'ANALYSE QUANTITATIVE DE LA SPERMATOGENESE DES POISSONS TÉLÉOSTÉENS
}

R. BILLARD, Aline SOLARI* et Anne-Marie ESCAFFRE

\author{
Laboratoire de Physiologie des Poissons, \\ * Station centrale de Physiologie animale, \\ Centre national de Recherches zootechniques, I. N. R. A., \\ 78350 Jouy en Josas
}

\begin{abstract}
RÉSUMÉ
Afin d'étudier quantitativement et rapidement la spermatogenèse des Poissons téléostéens nous avons évalué la part de volume occupée par les cellules germinales dans le testicule par la méthode des numérations ponctuelles (oculaire intégrateur I de Zeiss). On peut ainsi constater chez Carassius auratus des différentes dans la répartition des catégories cellulaires entre les parties antérieure médiane et postérieure. Cependant l'observation de 50 à 60 champs microscopiques sur une seule coupe transversale de la partie médiane du testicule, fournit un échantillon représentatif de la totalité de celui-ci.

La méthode d'échantillonnage ainsi mise au point a été appliquée sur des testicules d'animaux soumis à des traitements différents mais d'effets connus comme l'hypophysectomie seule ou suivie d'une supplémentation avec de la poudre hypophysaire brute de Carpe ou HCG. On montre ainsi que les volumes occupés par les cellules germinales rapportés au poids corporel permettent la discrimination nette entre les lots considérés ici et complètent l'information apportée par le rapport gonado-somatique.
\end{abstract}

\section{I. - INTRODUCTION}

Le rapport gonado-somatique dit RGS (poids de gonade exprimé en p. Ioo du poids corporel) est le critère le plus fréquemment employé dans les études de la spermatogenèse des Poissons. Un tel critère ne donne cependant qu'une idée globale et incomplète du phénomène. Le plus souvent l'analyse de la spermatogenèse est réalisée à partir d'un examen histologique permettant la description qualitative et quelquefois quantitative des types cellulaires présents dans le testicule. Les tentatives d'analyse quantitative sont peu nombreuses : les dénombrements cellulaires étant longs et souvent impossibles comme par exemple dans le cas des spermatozoides. 
Dans le cadre de nos études sur la spermatogenèse des Poissons téléostéens, nous avons recherché une technique d'analyse quantitative des différents types cellulaires, qui soit rapide et utilisable en routine. Cette technique, qui est exposée dans le présent travail, substitue au dénombrement des cellules germinales, une estimation de la part de volume testiculaire occupée par ces types cellulaires. Sa mise au point a consisté :

- A adapter la méthode des numérations ponctuelles pour l'estimation des volumes occupés par les cellules germinales dans le testicule.

- A rechercher l'échantillon le plus représentatif d'une coupe histologique pour un minimum de champs microscopiques observés.

- A vérifier si la méthode employée permet la discrimination d'animaux soumis à des traitements différents et d'effets connus comme l'hypophysectomie.

- A comparer l'information apportée par le RGS et le volume relatif occupé par les cellules du testicule.

\section{II. - PRÉSENTATION DE LA MÉTHODE DES NUMÉRATIONS PONCTUELLES}

\section{(A. SOLARI, I973)}

L'estimation des volumes relatifs des composants cellulaires dans un tissu, à partir de coupes histologiques d'épaisseur largement inférieure au diamètre cellulaire, repose sur l'équivalence entre la part de volume occupée par les cellules dans la coupe et la part de surface couverte par les sections de ces mêmes cellules sur la coupe. (Principe de Delesse.)

Soit :

$$
\mathrm{V}_{\mathrm{V}}=\mathrm{V}_{\alpha} / \mathrm{V}=\mathrm{A}_{\alpha} / \mathrm{A}=\mathrm{A}_{\mathrm{A}}
$$

Avec $\mathrm{V}$ et A : Volume et surface de la coupe

$\mathrm{V}_{\alpha}$ : Volume occupé par les cellules ( $\alpha$ ) dans la coupe

$A_{\alpha}$ : Surface occupée par les sections de cellules sur la coupe.

I,a méthode des numérations ponctuelles, pour 1'estimation des surfaces relatives $\mathrm{A}_{\alpha} / \mathrm{A}$, consiste à superposer un réseau de $\mathrm{P}$ points sur la coupe et à dénombrer les points $\mathrm{P}_{\alpha}$ sur la catégorie cellulaire à mesurer. Ce qui donne :

Soit :

$$
\begin{gathered}
\mathrm{A}_{\mathrm{A}}=\mathrm{A}_{\alpha} / \mathrm{A}=\mathrm{P}_{\alpha} / \mathrm{P} \\
\mathrm{V}_{\alpha} / \mathrm{V}=\mathrm{A}_{\alpha} / \mathrm{A}=\mathrm{P}_{\alpha} / \mathrm{P}
\end{gathered}
$$

Ceci permet d'obtenir le volume occupé par les cellules connaissant le volume de la coupe

$$
\mathrm{V}_{\alpha}=\mathrm{V} \cdot \mathrm{P}_{\alpha} / \mathrm{P}
$$

Le réseau employé (oculaire intégrateur I commercialisé par ZEISs) est composé de 25 points régulièrement espacés (fig. I). Pour les catégories cellulaires occupant une faible part de volume testiculaire, l'emploi de réseaux constitués d'un plus grand nombre de points, peu espacés entre eux, serait plus justifié. Cependant leur utilisation en microscopie optique entraîne une fatigue rapide de l'observateur et par suite 
des erreurs dans le dénombrement. Il est ainsi apparu plus judicieux d'augmenter le nombre de champs microscopiques à observer plutôt que le nombre de points par champ.

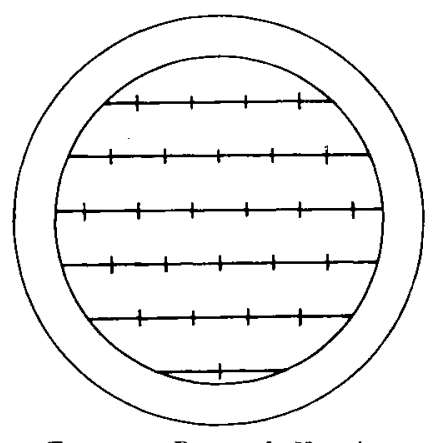

FIG. I. - Réseau de Henning

\section{III. - TECHNIQUES HISTOLOGIQUES}

La mise au point de la technique de comptage a été faite sur Carassius auratus et les résultats exposés dans cette étude se rapportent exclusivement à cette espèce. Après sacrifice des animaux par décapitation les testicules sont prélevés en totalité, pesés et fixés pendant 8 jours dans le mélange de Bouin-Hollande. Avant l'inclusion dans la paraffine le volume des gonades fixées est mesuré. Les types cellulaires retenus pour l'étude de la spermatogenèse sont les suivants:

\section{Espace intralobulaire}

Les spermatogonies $A\left(\mathrm{G}_{\mathrm{A}}\right)$ (diamètre moyen $\mathrm{I} 3,4 \mu$ ): il s'agit de toutes les spermatogonies non regroupées en cystes et qui sont caractérisées par leur grande taille et dont le noyau d'aspect clair est coloré par l'orangé $\mathrm{G}$.

Les spermatogonies $B\left(\mathrm{G}_{\mathrm{B}}\right)$ (diamètre moyen $7 \mu$ ): il s'agit de toutes les générations de spermatogonies contenues à l'intérieur de cystes. Les cellules sont plus petites, le cytoplasme et la chromatine sont beaucoup plus intensément colorés que dans les spermatogonies $\mathrm{A}$.

Les spermatocytes $I$ (Spcytes) (diamètre moyen $7,7 \mu$ ): Les étapes successives de la prophase méíotique, qui se déroulent également à l'intérieur de cystes, sont faciles à identifier et à compter (leptotène, zygotène, pachytène), mais dans cette étude ces types cellulaires sont regroupés.

Les spermatocytes II et les spermatides (Sptides) (diamètre moyen 3,6 $\mu$ ): Les spermatocytes II sont peu fréquents et difficiles à distinguer des spermatides. Ces deux catégories cellulaires, elles aussi organisées en cystes, sont regroupées lors des comptages.

Les spermatozoïdes (spz) : Les spermatozoïdes se concentrent dans la lumière des lobules séminifères. Chez le Carassin, la taille de la tête spermatique, de forme à peu près sphérique, est d'environ $2,3 \mu$. 
Les cellules de Sertoli (S) (I3,5 $\mu$-I5 $\mu$ ) : Ces cellules somatiques dont la forme varie au cours de la spermatogenèse sont localisées autour des lobules testiculaires (BILLARD et al., I972).

La lumière des lobules non occupée par des spermatozoïdes (L). Ce paramètre rend compte de l'état de dilatation des lobules dû à la sécrétion du " liquide séminal " par les cellules de Sertoli.

\section{Espace extralobulaire (VS)}

Il existe dans l'espace extralobulaire :

- Les vaisseaux sanguins ;

- Les cellules conjonctives ;

- Les cellules interstitielles.

Les vaisseaux sanguins sont faciles à identifier mais du fait d'une répartition non aléatoire, des travaux préliminaires ont montré qu'il était nécessaire, pour obtenir une précision suffisante, de réaliser un nombre de comptages trop élevé pour satisfaire aux objectifs de rapidité demandés à la méthode.

Il n'est pas possible de distinguer avec certitude les cellules conjonctives des cellules interstitielles, en dehors de tests histochimiques. Ces tests étant d'application difficile dans une technique de routine, les cellules interstitielles et le conjonctif extralobulaire sont regroupés avec les vaisseaux sanguins.

Compte tenu de la taille réduite des cellules germinales, les exigences de la méthode des numérations ponctuelles (cf II) nous imposeraient une épaisseur de coupe inférieure à $2 \mu$. Cependant, l'emploi de coupes de $5 \mu$ d'épaisseur se trouve justifié ici car l'intérêt de l'observation ne se porte pas sur les cellules individuelles — à l'exception des spermatogonies $\mathrm{A}$ et des Sertoli dont le diamètre est supérieur à ro $\mu$ - mais sur les cystes et sur l'agrégat des spermatozoïdes dans la lumière.

La coloration a été faite par l'hématoxyline ferrique de Régaud, orange $G$, bleu d'aniline.

La mise au point de la méthode d'échantillonnage a débuté par une étude de la répartition spatiale de types cellulaires dans le testicule.

\section{IV. - RÉPARTITION DES CELLULES SUR COUPES SAGITTAIES DE, TESTICULES}

Dans les testicules de téléostéens la répartition des types cellulaires n'est pas homogène : un simple examen microscopique d'une coupe longitudinale montre que les premiers stades de la spermatogenèse sont mieux représentés dans la partie antérieure que dans la partie postérieure qui est quelquefois stérile. A titre de vérification une coupe longitudinale sagittale passant par le canal déférent a été observée en 3 zones, délimitées selon la figure 2 .

Les pourcentages de points qui se superposent aux différentes catégories cellulaires (tabl. I) diffèrent significativement entre les 3 zones $(\mathrm{P}<\mathrm{I}$ p. Ioo). La zone III présente une plus grande quantité de spermatozoïdes mais peu de spermatocytes $I$, de spermatides et de spermatogonies $B$ par rapport aux 2 autres zones plus antérieures. Ces 2 dernières semblent comparables entre elles, cependant dans la zone II (zone 


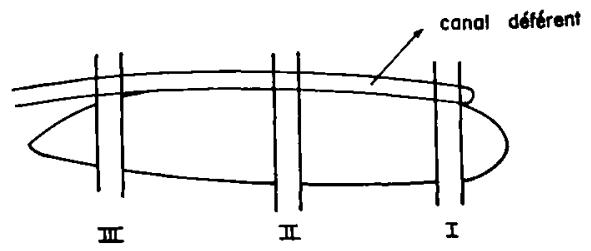

FrG. 2. - Coupe longitudinale shematique de testicule de Carassin

$$
\begin{aligned}
& \text { I : partie antérieure } \\
& \text { II : partie médiane } \\
& \text { III : partie postérieure }
\end{aligned}
$$
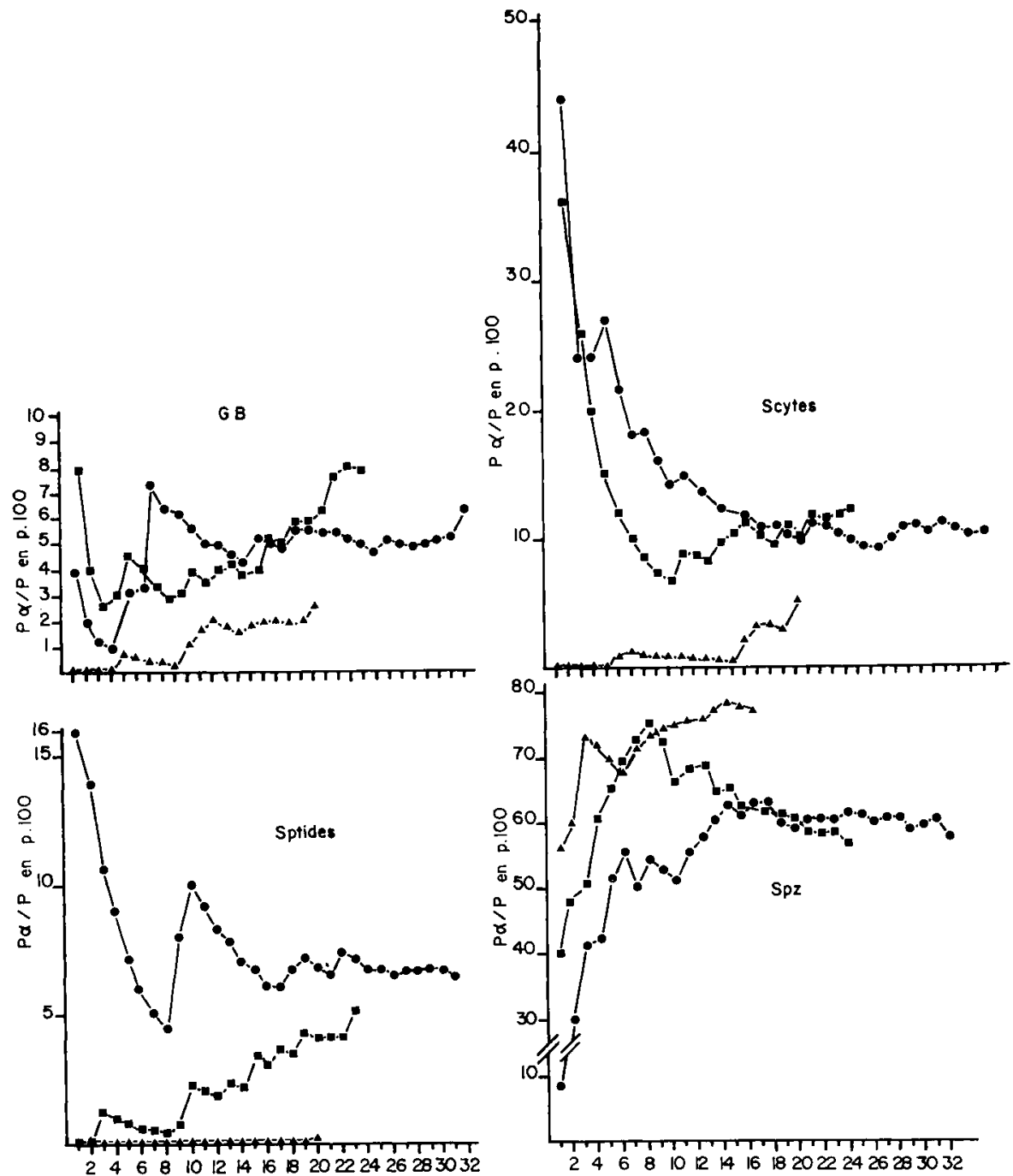

$24 \quad 6 \quad 8 \quad 101214161820222426283032$

$246810 R 14161820222426283032$

nombre de champs

Fig. 3. - Variation du pourcentage de points en fonction du nombre de champs microscopiques observés par zone

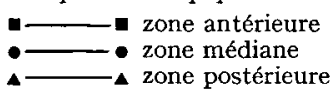


médiane) la variation du rapport $\mathrm{P}_{\alpha} / \mathrm{P}$ en fonction du nombre de champs observés se stabilise plus rapidement à partir $\mathbf{d u} \mathbf{I}^{\mathrm{e}}, 20^{\mathrm{e}}$ champ microscopique et ceci pour toutes les catégories cellulaires (fig. 3). En conséquence dans la suite de l'étude l'analyse portera sur des coupes transversales de la zone médiane du testicule.

\section{TABLEAU I}

Pourcentage de points $\left(\mathrm{P}_{\alpha} / \mathrm{P} \times\right.$ Ioo)

se superposant aux différentes catégories cellulaires pour chaque zone étudiée

\begin{tabular}{c|c|c|c|c|c|c|c|c|c|c}
\hline \hline $\begin{array}{c}\text { Zone du } \\
\text { testicule }\end{array}$ & $\begin{array}{c}\text { Espace } \\
\text { extra- } \\
\text { lobulaire }\end{array}$ & Sertoli & $\mathrm{G}_{\mathbf{A}}$ & $\mathrm{G}_{\mathbf{B}}$ & Spcytes & Sptides & Spz & Pycnose & Lumière & $\begin{array}{c}\text { Nbre } \\
\text { de } \\
\text { champs }\end{array}$ \\
\hline $\begin{array}{c}\text { Antérieure } \\
\text { I }\end{array}$ & $\mathbf{5 , 4}$ & 7,8 & 1,9 & 8,0 & 12,0 & 5,2 & 56,9 & 1,2 & 1,6 & 23 \\
\hline $\begin{array}{c}\text { Médiane } \\
\text { II }\end{array}$ & 4,8 & 7,0 & 2,7 & 6,5 & 10,3 & 6,5 & 59,1 & 2,1 & $\mathbf{1 , 2}$ & 31 \\
\hline $\begin{array}{c}\text { Postérieure } \\
\text { III }\end{array}$ & 5,4 & 5,2 & 1,8 & 2,6 & 5,0 & 0 & 76,0 & 0,4 & 3,6 & 20 \\
\hline
\end{tabular}

\section{V. - RÉPARTITION DES CELLULES SUR COUPES TRANSVERSALES DE LA ZONE MÉDIANE DU TESTICULE}

L'analyse porte sur 3 animaux, chacun étant représenté par une seule coupe transversale de la zone médiane du testicule. L'observation de la coupe est effectuée à partir de champs microscopiques adjacents, disposés en lignes séparées d'un intervalle constant de 4 champs, dans le sens dorso-ventral (fig. 4).

Sur les 3 animaux, il a été observé respectivement II3, I05 et I47 champs microscopiques (tabl. 2 ) ; les pourcentages moyens de points dénombrés sur chaque catégorie cellulaire seront considérés comme représentatifs de la coupe et serviront de référence dans la recherche d'une méthode rapide d'échantillonnage. Celle-ci tiendra

TABLEAU 2

Nombre total de champs microscopiques observés par animal

\begin{tabular}{c|c|c|c}
\hline $\begin{array}{c}\text { Numéro } \\
\text { de l'animal }\end{array}$ & $\begin{array}{c}\text { Nombre de lignes } \\
\text { d'observation }\end{array}$ & $\begin{array}{c}\text { Nombre } \\
\text { de champs }\end{array}$ & $\begin{array}{c}\text { Nombre total } \\
\text { de points }\end{array}$ \\
\hline 1 & 11 & 113 & 2825 \\
2 & 9 & 105 & 2625 \\
3 & 9 & 147 & 3675 \\
\hline
\end{tabular}



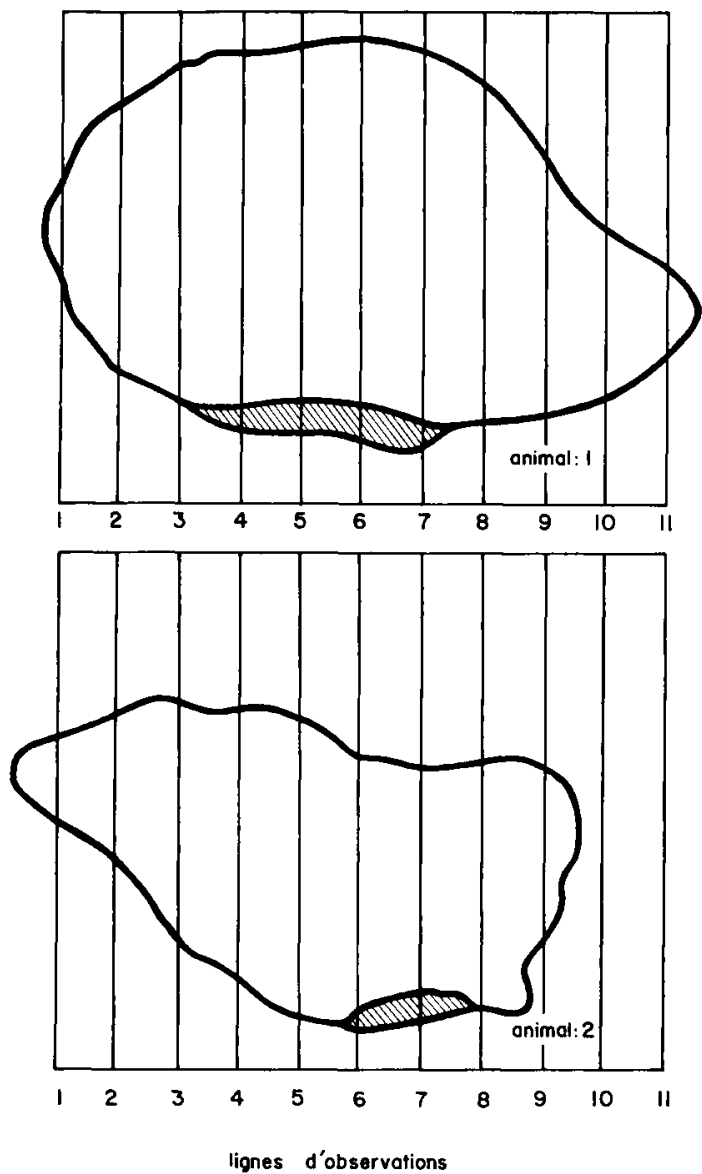

FIG. 4. - Schéma de la coupe transversale médiane de testicule des animaux 1 et 2 Les lignes d'observations représentent la position des champs microscopiques sur la coupe. Zone hachurée: canal déférent

compte de la répartition hétérogène des cellules sur la coupe (fig. 5) sauf pour les spermatogonies A chez les animaux $n^{\circ} 2$ et $n^{\circ} 3$ et les Sertoli chez les 3 animaux (comparaison des pourcentages moyens par ligne ; méthode du $\mathrm{X}^{2}$ ).

I. - Détermination de la zone de la coupe et du nombre minimum de champs microscopiques à observer

L'approximation du nombre $n$ de mesures qu'il est nécessaire de réaliser pour estimer une valeur moyenne avec une précision donnée n'a pu être calculée par la relation statistique classique :

$$
n=t_{\alpha}^{2} s^{2} / \mathrm{d}^{2}
$$

ou $t_{\alpha}$ : variable de Student au seuil $\alpha$ pour $(n-\mathrm{I}) \mathrm{dl}$;

$s^{2}$ : estimation de la variance des mesures ;

$\mathrm{d}$ : erreur maximum consentie.

Annales de Biologie animale. - 1974. 
94

R. BILLARD, A. SOLARI, A.-M. ESCAFFRE

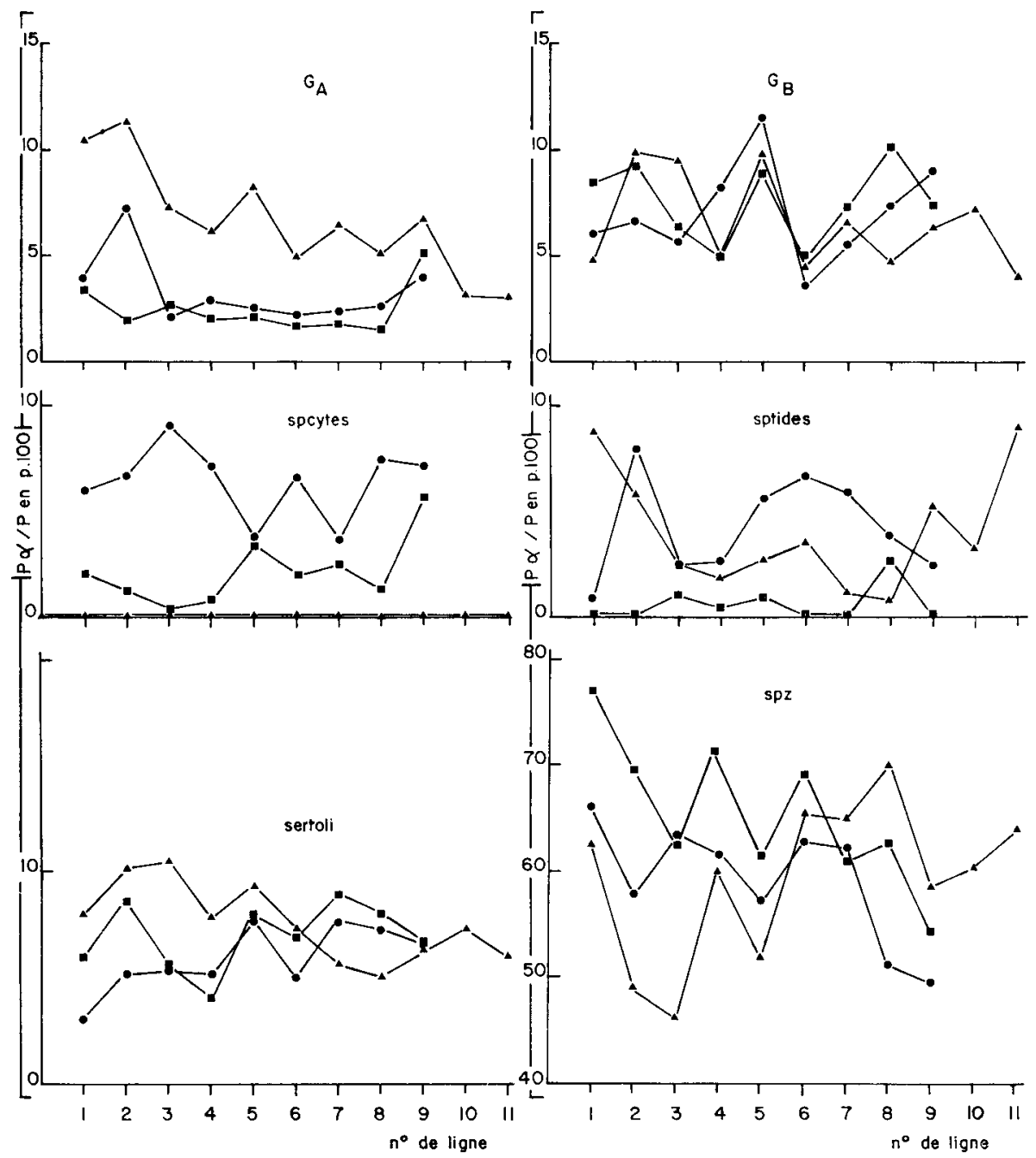

FIG. 5. - Variation du pourcentage de points en fonction du numéro de ligne d'observation

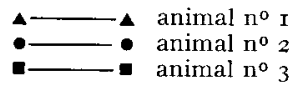


Celle-ci implique l'assimilation du pourcentage moyen $\left(\mathrm{P}_{\alpha} / \mathrm{P} \times\right.$ roo $)$ à une variable normale et suppose la répartition au hasard sur la coupe histologique des cystes et agrégats cellulaires ainsi que des champs microscopiques. Il est toujours possible de satisfaire à la première condition $\left(\mathrm{P}_{\alpha} / \mathrm{P} \times\right.$ IOo - variable normale $)$ en augmentant suffisamment le nombre de champs microscopiques mais ceci supprimerait l'intérêt espéré de la méthode : la rapidité. Les comparaisons des résultats moyens par ligne conduisent au rejet de l'hypothèse d'une répartition homogène des différentes catégories cellulaires. Quand à l'échantillonnage au hasard - pour des considérations pratiques - il est rarement utilisé dans l'exploration microscopique contrairement au balayage systématique (observation d'un champ tous les $k$ champs ou d'un groupe de champs tous les $k$ groupes). Il convient donc de rechercher empiriquement le nombre de lignes - formées de champs microscopiques adjacents - qu'il suffit d'observer pour réaliser un échantillonnage représentatif de la coupe transversale.

\section{a) Constitution de différents échantillons par animal}

A partir de l'observation systématique des coupes transversales, des échantillons, comprenant un nombre moindre de lignes, sont constitués et leurs résultats comparés aux valeurs de référence $\pm 5 \mathrm{p}$. Ioo.

Pour l'animal $n^{\circ} \mathrm{I}$, un ensemble de 7 échantillons positionnés différemment sur la coupe et comprenant un nombre variable de champs microscopiques (tabl. 3)

\section{TABLEAU 3}

Description des 7 échantillons prélevés sur la coupe transversale de testicule de l'animal $n^{\circ} 1$

\begin{tabular}{|c|c|c|}
\hline $\begin{array}{l}\text { Numéro de } \\
\text { l'échantillon }\end{array}$ & $\begin{array}{l}\text { Numéro des lignes } \\
\text { constituant l'échantillon }\end{array}$ & $\begin{array}{l}\text { Nombre } \\
\text { de champs }\end{array}$ \\
\hline 1 & $\begin{array}{l}\text { de } 1 \text { à } 6 \text { représentant } \\
\text { ainsi la moitié de la coupe }\end{array}$ & 66 \\
\hline 2 & de la 1re à la $5^{\text {e }}$ & 50 \\
\hline 3 & de la 1 re à la $4 \mathrm{e}$ & 38 \\
\hline 4 & $\begin{array}{l}4 \text { lignes prélevées au hasard } \\
\qquad(4-7-9-10)\end{array}$ & 44 \\
\hline 5 & $1-2-10-11$ & 30 \\
\hline 6 & $4-3-8-9$ & 42 \\
\hline 7 & $4-5-6-7$ & 52 \\
\hline
\end{tabular}

permettra de délimiter une zone représentative. C'est exclusivement dans cette zone

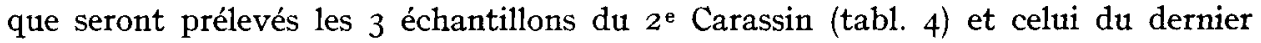
animal. 


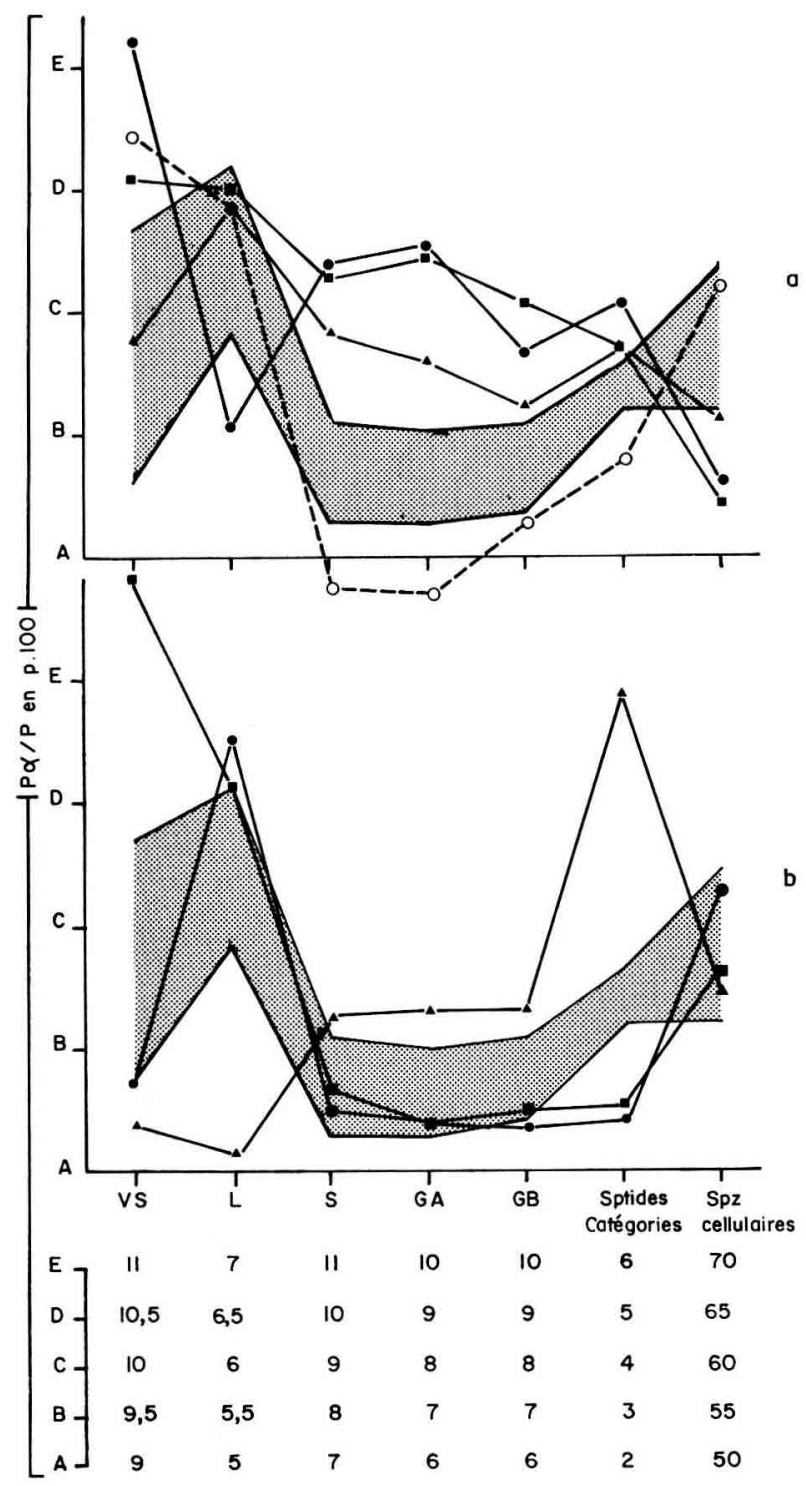

FIG. 6. - Pour l'animal no 1 comparaison des différentes méthodes d'échantillonnages Zone pointillée : valeurs de référence $\pm 5 \mathrm{p}$. Ioo

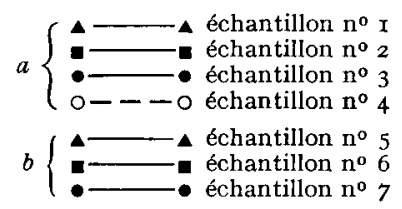




\section{TABLEAU 4}

Les différents échantillons prélevés

dans la zone du canal déférent d'une coupe transversale de testicule de l'animal n० 2

\begin{tabular}{|c|c|c|}
\hline $\begin{array}{l}\text { Numéro de } \\
\text { l'échantillon }\end{array}$ & $\begin{array}{l}\text { Numéro des lignes } \\
\text { constituant l'échantillon }\end{array}$ & Nombre de champs \\
\hline 1 & 7 et 6 & 27 \\
\hline 2 & $5-7-6-8$ & 60 \\
\hline 3 & $4-5-6-7$ & 58 \\
\hline
\end{tabular}

b) Comparaison des échantillons.

La comparaison des résultats des échantjllons aux valeurs de référence montre que seuls les 2 derniers échantillons, parmi les 7 étudiés chez l'animal $n^{\circ} \mathrm{I}$, peuvent être considérés comme représentatifs de la coupe (fig. $6 a$ et $6 b$ ).

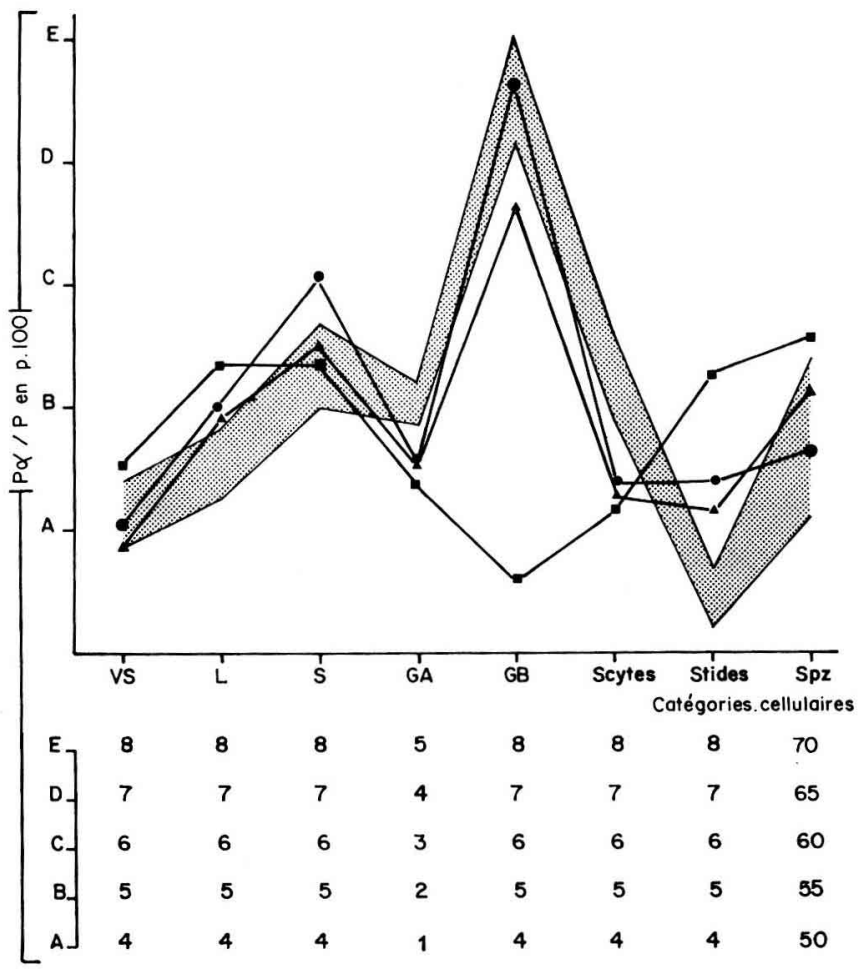

FIG. 7. - Pour l'animal no 2 comparaison de 3 échantillons prélevés dans la zone du canal déférent

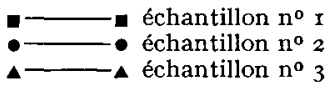


R. BILIARD, A. SOLARI, A.-M. ESCAFFRE

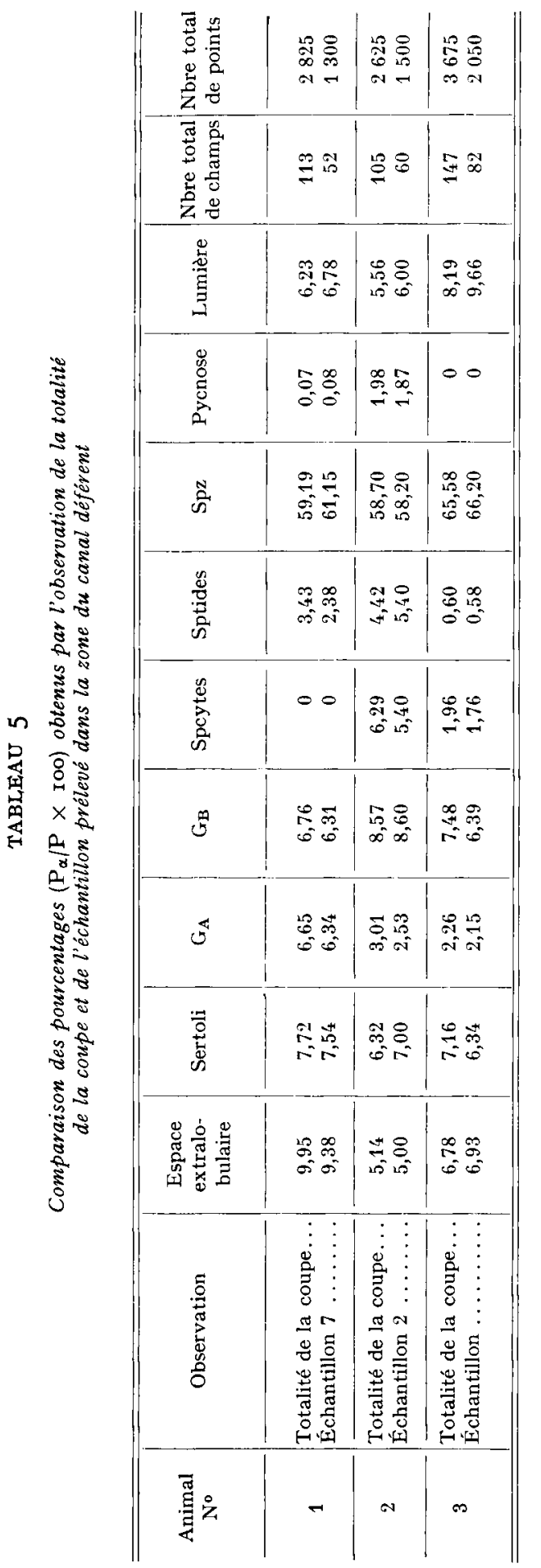

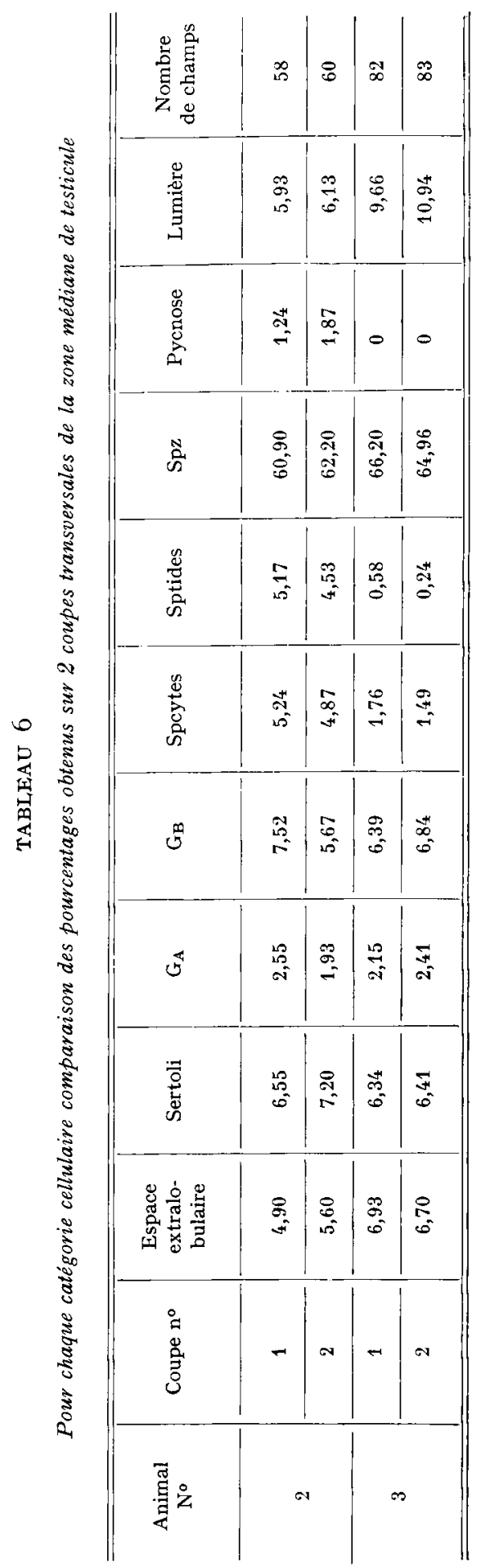


Ils comprennent respectivement 42 et $5^{2}$ champs microscopiques situés dans la zone passant par le canal déférent. Le dernier échantillon $\left(\mathrm{n}^{0} 7\right)$ à l'avantage d'être facilement repérable par l'observation puisque les lignes d'observations passent par l'axe du canal déférent (fig. 4). En conséquence, pour cet animal, la répartition en ligne, dans la zone du canal déférent, d'environ 40-50 champs microscopiques apporte la même information que le balayage systématique de la coupe.

Ce résultat se trouve confirmé chez l'animal $n^{\circ} 2$ pour les échantillons 2 et 3 comprenant respectivement 60 et 58 champs (fig. 7). Le désaccord entre les valeurs de référence et celles fournies par l'échantillon $\mathrm{n}^{\circ} \mathrm{I}$ principalement pour 2 catégories fondamentales dans l'étude de la spermatogenèse (Gonies B et spermatides) s'explique par le nombre faible $(n=27)$ de champs microscopiques observés (fig. 7).

Pour les 3 animaux, la concordance entre les valeurs de référence et les pourcentages obtenus à partir d'échantillons situés dans la zone du canal déférent, (tabl. 5) permet d'envisager la diminution de $50 \mathrm{p}$. Ioo du nombre de champs microscopiques à observer par coupe.

En conclusion, chez le Carassin, l'échantillon le plus représentatif d'une coupe transversale de testicule doit être constitué de 50 ou 60 champs microscopiques, répartis dans la zone du canal déférent.

\section{2. - Nombres minimum de coupes à observer dans la zone médiane du testicule}

L'homogénéité des pourcentages (Ioo $\mathrm{P}_{\alpha} / \mathrm{P}$ ) obtenus sur des coupes espacées de $60 \mu$ et situées dans la zone médiane du testicule (tabl. 6) montre qu'il suffit d'observer une seule coupe par animal ce qui permet d'augmenter le nombre d'animaux en expérience.

\section{VI. — GÉNÉRALISATION}

\section{COMPARAISON DE PLUSIEURS ANIMAUX ENTRE EUX}

La méthode qui vient d'être exposée revient à quantifier les différents types cellulaires par leur rapport en volume. Ce mode d'expression est suffisant lorsqu'il s'agit de comparer des animaux au même stade de développement spermatogénétique et de même poids testiculaire. Ce système de représentation a l'avantage de permettre une exploitation statistique des résultats en individualisant chaque champ microscopique (SoLARI, I973).

Cependant le poids testiculaire varie de façon considérable soit pour un même animal en fonction de la saison, soit d'un animal à l'autre. Il devient alors nécessaire d'obtenir une représentation de la quantité de cellules présentes dans la totalité des testicules. Considérant la relation (3) il suffit d'étendre le rapport au volume total des testicules et la généralisation de la méthode donne :

$$
\mathrm{V}_{\alpha}=\left(\mathrm{P}_{\alpha} / \mathrm{P}\right) \cdot \mathrm{V}_{\mathbf{T}}
$$

$\mathrm{V}_{\mathbf{T}}$ : Volume total des testicules après fixation $\left(\mathrm{en}^{\mathrm{mm}} \mathrm{m}^{3}\right.$;

$\mathrm{P}_{\alpha}$ : Nombre de points se superposant à la catégorie cellulaire $\alpha$;

$\mathrm{P}$ : Nombre total de points comptés ;

$\mathrm{V}_{\alpha}$ : Volume occupé par la catégorie cellulaire $\alpha\left(\mathrm{en} \mathrm{mm}^{3}\right)$. 
Pour permettre la comparaison entre animaux de poids différents et afin d'éliminer la relation volume des gonades-poids du corps, il suffit de ramener $\mathrm{V}_{\alpha}$ au gramme de poids corporel.

\section{VII. - APPLICATION : COMPARAISON \\ DE LA SPERMATOGENÈSE D'ANIMAUX INTACTS}

\section{ET HYPOPHYSECTOMISÉS}

\section{A. - Matériel et méthodes}

\section{Techniques d'hypophysectomie et de supplémentation hormonales.}

Les Carassins dont le poids moyen est de $50 \mathrm{~g}$ sont répartis par groupe de 5 à ro dans des aquariums de $50 \mathrm{l}$ avec de l'eau ordinaire pour les mâles témoins (lot $\left.\mathrm{H}^{+}\right)$et de l'eau salée $(0,8 \mathrm{p}$. Ioo de CLNa) pour les mâles hypophysectomisés.

L'hypophysectomie est pratiquée par voie operculaire (CHAvin, I956; modifié par LaHLou et SAWYER, 1969).

Le traitement de supplémentation débute 24 heures après hypophysectomie et comporte l'administration de poudre hypophysaire acétonique de Carpe (ro $\mu \mathrm{g} / \mathrm{g}$ de poids vif/injection : lot $\mathrm{H}^{-}+$Carpe) et de $\mathrm{HCG}$ (Io UI/g de poids vif/injection : lot $\mathrm{H}^{-}+\mathrm{HCG}$ ). Un $3^{\mathrm{e}}$ lot ne reçoit que le solvant (liquide physiologique à $8 \mathrm{p}$. I ooo lot $\mathrm{H}^{-}$). Les animaux reçoivent au total Io injections à raison de 3 injections par semaine (lundi, mercredi, vendredi) et sont élevés à la température de $17^{\circ} \mathrm{C}$ et sous photopériode $12 \mathrm{~L}-\mathrm{I}_{2} \mathrm{~N}$. L'expérience s'est déroulée en septembre et octobre 1969 .

\section{Technique statistique.}

L'effet des traitements sur le volume total occupé par les cellules germinales rapporté au poids du corps et sur le RGS a été testé par des méthodes non paramétriques (SIEGEL, 1956).

Les intervalles de confiance non paramétriques sont calculés à partir des tables de Tukey (BLIss, I967).

$\mathrm{L}^{\prime}$ analyse statistique porte sur 5 animaux pour Ies lots $\mathrm{H}^{+}, \mathrm{H}^{-}$et $\mathrm{H}^{-}+$hypophyse de Carpe et sur 8 animaux pour le lot $\mathrm{H}^{-}+\mathrm{HCG}$.

\section{B. - Effet des traitements}

I. Sur les volumes occupés par les cellules germinales (tabl. 7, fig. 8).

La méthode de quantification de la spermatogenèse proposée, permet d'apprécier les effets des traitements (hypophysectomie et supplémentation gonadotrope). L'ablation de l'hypophyse supprime au bout de quelques jours les spermatogonies B, les spermatocytes, les spermatides et les spermatozoïdes, résultats déjà obtenus sur d'autres animaux et par d'autres techniques de mesure.

Ces catégories cellulaires peuvent être régénérées par un traitement avec de la poudre hypophysaire brute de Carpe mais pas par HCG. Ce dernier lot s'apparente au groupe d'animaux hypophysectomisés sauf pour les spermatozoïdes qui ont été maintenus dans la lumière des lobules. Les quantités de spermatogonies A et de cellules de Sertoli n'ont pas été affectées par les traitements.

\section{Effet des traitements sur le rapport gonado-somatique (tabl. 8).}

Les 4 lots diffèrent significativement entre eux (fig. 9). La différence est particulièrement nette entre les animaux intacts et hypophysectomisés $(P<0,008)$. 


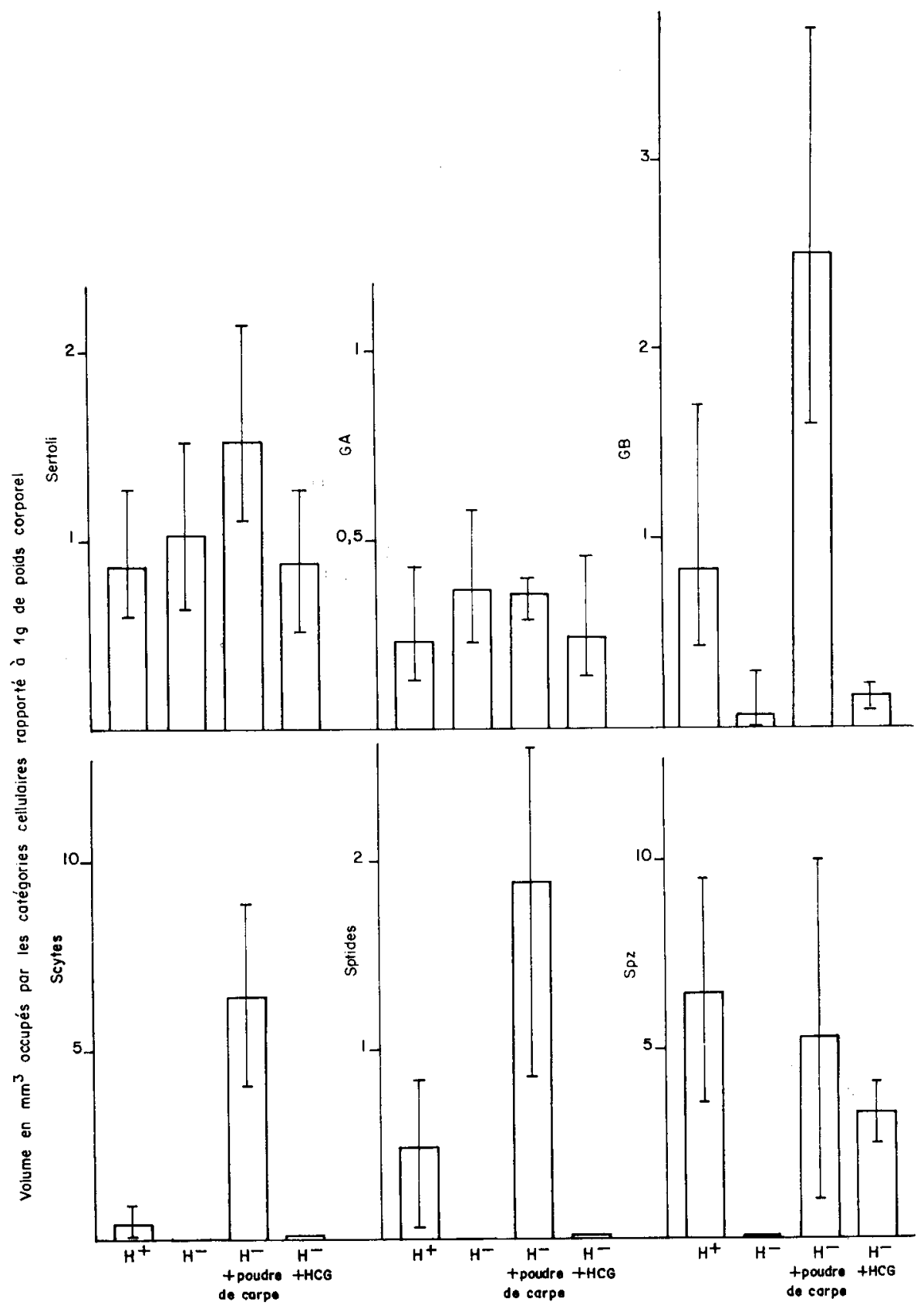

FIG. 8. - Par lot expérimental, intervalle de confiance non paramétrique au seuil 10 p. 100 pour chaque catégorie cellulaire 
TABLEAU 7

Résultats des tests de comparaison des 4 lots expérimentaux

Les 4 lots sont homogènes pour les Sertoli et spermatogonies $\mathrm{A}$

Les 4 lots diffèrent significativement entre eux pour les 4 catégories cellulaires suivantes.

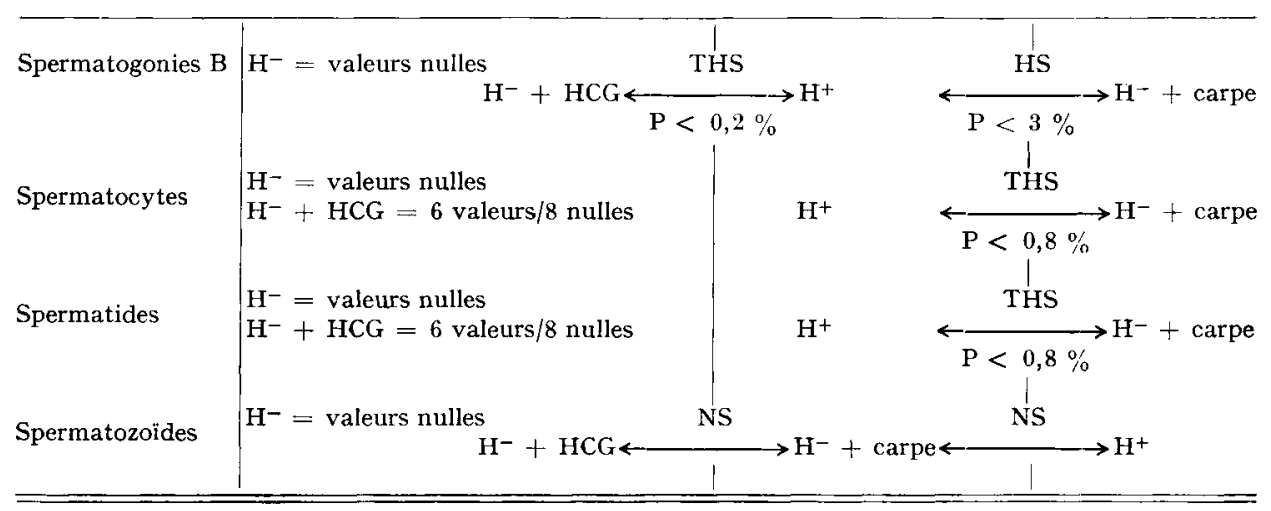

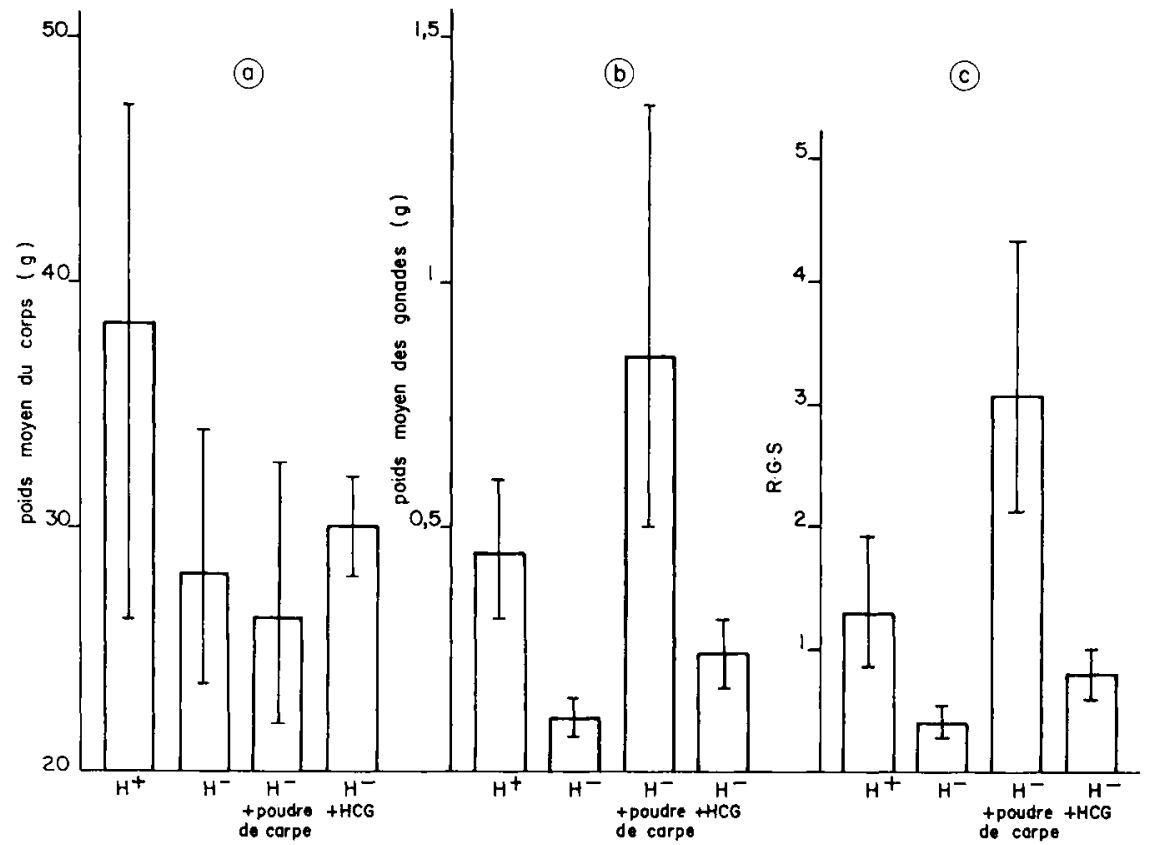

FIG. 9. - Intervalle de confiance non paramétrique au seuil 10 p. 100 pour le $R G S$ moyen 
TABLEAU 8

Valeurs moyennes des RGS après supplémentations hormonales chez Carassius auratus comparées aux animaux témoins intacts et hypophysectomisés

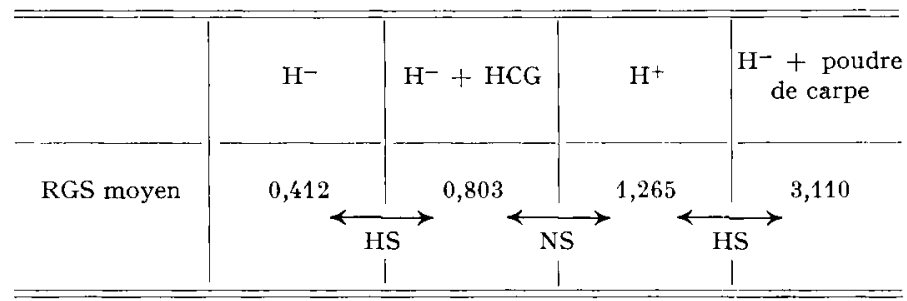

La supplémentation avec la poudre de Carpe se traduit par une forte élévation du poids testiculaire puisque les animaux diffèrent non seulement des hypophysectomisés $(\mathrm{P}<0,004)$ mais aussi des témoins $\mathrm{H}^{+}(\mathrm{P}<0,0 \mathrm{I} 6)$. L'effet de HCG est plus modéré ; les RGS bien que supérieurs à ceux du lot $\mathrm{H}^{-}(\mathrm{P}<0,003)$ sont semblables aux valeurs trouvées chez les animaux témoins $(P<0, I 0)$.

\section{VIII. - CONCLUSION}

La méthode d'analyse quantitative de la spermatogenèse par estimation du volume occupé par les différents types de cellules dans les testicules présente un certain nombre d'avantages.

- La rapidité de mise en ouvre (examen par animal de 50 champs microscopiques sur lesquels 25 points sont à dénombrer chaque fois) en fait une technique de routine.

- En l'absence de structures unitaires stables dans les testicules de poissons téléostéens à cycle reproducteur annuel (comme par exemple une coupe orthogonale de tubule séminifère de vertèbré supérieur) cette méthode permet d'inventorier l'ensemble des cellules de la totalité des 2 testicules de chaque animal. Il devient alors possible de comparer des individus de stade et de poids différents.

- Cette méthode qui a d'abord été mise au point chez le Carassin et dont les détails de l'échantillonnage viennent d'être exposés a également été mise en œuvre chez la Truite (BILLARD et LugueT, I974) la Carpe et le Gardon et semble généralisable à l'ensemble des poissons téléostéens.

Cette méthode utilisée au laboratoire depuis plusieurs années a permis de conduire un certain nombre d'études sur des problèmes d'endocrinologie de la spermatogenèse et sur l'évolution de la spermatogenèse au cours du cycle annuel. 


\section{SUMMARY \\ TECHNOLOGICAL, NOTE ON A METHOD OF QUANTITATIVE ANALYSIS \\ FOR TELEOST FISH SPERMATOGENESIS}

In order to quantitatively and rapidly study spermatogenesis of teleost fishes, we estimated the amount of volume occupied by germ cells in the testicle by the punctual numeration method (Zeiss ocular integrator I). Repartition of cell types is different in the anterior median or posterior parts of the testicle of Carassius auratus. However observation of a single transversal cross-section, using 50 or 60 microscopic fiels scattered in the median part of the testicle, gives a representative sample of the testicle.

This improved sampling method was applied to testicles of animals undergoing different treatments of known effect, such as hypophysectomy alone or followed by supplementation with crude carp or HCG pituitary powder.

The volumes occupied by germ cells are divided by body weight, thus allowing a clear distinction between the lots considered here and completing information from the gonadosomatic index.

\section{RÉFÉRENCES BIBLIOGRAPHIQUES}

Billard R., Jalabert B., Breton B., i972. Les cellules de Sertoli des Poissons Téléostéens. Ann. Biol. anim. Bioch. Biophys., 12, 19-32.

BILlaRd R., Luquet P., I974. Le cycle spermatogénétique de la Truite. (En préparation).

Bliss C. I., I967. Statistics in biology, volume I, page 201-203 Mc. Graw-Hill Book Company. New York.

Chavin W., 1956. Pituitary adrenal control of melanization in Xanthic goldfish Carassius auratus L. J. Exp. Zool., 133, I-36.

LALHOU B., SAWYER W., r969. Electrolyte balance in hypophysectomized goldfish Carassius auratus L. Gen. Comp. Endocrinol., 12, 370-377.

SIEGEL S., 1956. Nonparametric statistics for the behavioral sciences, 312, Mc. Graw-Hill Book Company New York.

Solari A., 1973. Étude quantitative d'organes et de tissus. I. Méthodes d'estimation des volumes. Ann. Biol. anim. Bioch. Biophys. 13, 247-265. 